\title{
The profiles of public and private patients in maternal healthcare: a longitudinal study to examine adverse selection
}

\author{
Jananie William ${ }^{1 *}$ (D) Bronwyn Loong ${ }^{1}$, Catherine Chojenta ${ }^{2}$ and Deborah Loxton ${ }^{2}$ \\ ${ }^{1}$ Research School of Finance, Actuarial Studies and Statistics, Australian National University, Australia 2601 and ${ }^{2}$ Research \\ Centre for Generational Health \& Ageing, The University of Newcastle, Australia 2308 \\ *Corresponding author. Email: jananie.william@anu.edu.au
}

(Received 15 January 2019; revised 30 April 2019; accepted 13 May 2019)

\begin{abstract}
In this article, we investigate differences in the profiles of patients within the Australian mixed publicprivate maternal health system to examine the extent of adverse selection. There are conflicting influences on adverse selection within the private health sector in Australia due to government regulations that incentivise lower risk segments of the population to purchase community-rated private health insurance. We use a two-phase modelling methodology that incorporates statistical learning and logistic regression on a dataset that links administrative and longitudinal survey data for a large cohort of women. We find that the key predictor of private patient status is having private health insurance, which itself is largely driven by sociodemographic factors rather than health-or pregnancy-related factors. Additionally, transitioning between the public-private systems for a subsequent pregnancy is uncommon; however, it is primarily driven by changes in private health insurance when it occurs. Other significant factors when transitioning to the private system for a second pregnancy are hypertension, increased access to specialists and stress related to previous motherhood experiences. Consequently, there is limited evidence of adverse selection in this market, with targeted financial incentives likely outweighing the impact of community rating even during childbearing years where private health service use increases.
\end{abstract}

Keywords: Private health insurance; maternal health; adverse selection

\section{Introduction}

Australia's maternal healthcare system is composed of mixed public-private service provision and funding arrangements. The overall system is characterised by a predominantly obstetricianled private system, and a midwife-led or shared-care model with general practitioners in the public system. There are other models of care available in the public system which include the involvement of multidisciplinary obstetric teams if indicated by risk factors or complications (Adams et al., 2018). Public-private comparisons have shown that there are clear disparities in cost risk factors (William et al., 2018b) and clinical services (Roberts et al., 2000; Shorten \& Shorten, 2000; Dahlen et al., 2012, 2014; Einarsdóttir et al., 2012, 2013b; Nippita et al., 2015) with mixed results on how this may impact on neonatal and maternal outcomes (Shorten \& Shorten, 2002; Robson et al., 2009; Einarsdóttir et al., 2012, 2013a,b,c; Dahlen et al., 2014; Adams et al., 2017, 2018; Jang et al., 2017) by system; however, these studies have not investigated the detailed profiles of patients that enter into each system. Furthermore, there have been no studies that directly address the potential for adverse selection in private maternal health services, 
despite the substantially increased usage of private health insurance (PHI) during childbearing years for women (Australian Prudential Regulatory Authority, 2018). In particular, previous studies do not determine whether women who are at higher risk of pregnancy complications are more likely to take-up PHI and elect private services; or whether high-risk women transition between systems for subsequent pregnancies. A better understanding of the profiles of women in each system will help infer whether adverse selection is evident in the context of this maternal health system.

In Australia, all residents are entitled to free hospital services in the public system under Medicare, a universal government-funded health insurance scheme. All individuals can also elect to use the private system, but the cost-sharing arrangements for the private sector are more complex and likely to involve a combination of individual, government and PHI sources. Individuals with PHI may use this insurance to help fund private services and individuals without PHI largely self-fund private care. It is unlikely that PHI will wholly cover the cost of private hospital services during the perinatal period, so the individual is likely to face out-of-pocket expenses when using private services. The regulation of the Australian system creates an additional layer of complexity in that financial penalties are used to encourage certain groups to purchase PHI. Specifically, the Medicare Levy Surcharge (MLS; introduced in 1997) applies as an additional tax to high-income earners that do not have PHI for hospital treatment and the Lifetime Health Cover (LHC; introduced in 2000) applies a premium loading to individuals who do not purchase PHI before the age of 30 years (Buchmueller, 2008; Australian Taxation Office, 2018a,b; The Department of Health, 2018a). A government-funded premium subsidy was also introduced with the MLS to make PHI more affordable and encourage more people into the private system (Australian Taxation Office, $2018 \mathrm{~b}$ ). While these policies may have encouraged $45 \%$ of the population to purchase PHI in 2018 (Australian Prudential Regulatory Authority, 2018) and 26\% of all hospital births to occur in private hospitals in 2014 (Australian Institute of Health and Welfare, 2018), those with PHI may still elect to be treated as public patients for free under Medicare and thereby avoid any out-ofpocket expenses. Further details of the Australian maternal health system are provided elsewhere (William et al., 2018a,b).

Adverse selection has been widely studied in the insurance literature, and it is well recognised that information asymmetry between insurers and individuals could lead to adverse selection (Hoy \& Lun, 2017; Thomas, 2017). In other words, the potential for individuals to have more information about their own risk profile which is not observable to insurers could lead to higher risks being more likely to purchase the insurance (and conversely, lower risks being less likely to purchase insurance). For PHI specifically, adverse selection may arise if it attracts individuals who believe they are at higher risk of health problems, or in the case of maternal health, at higher risk of pregnancy-or birth-related complications. Although most insurers mitigate the pregnancy risk to some extent by applying a 12-month waiting period to pregnancy-related expenses, there still remains uncertainty as to how high-risk women navigate the public-private maternal health system, particularly for subsequent pregnancies. In addition to these general market observations, PHI is community-rated in Australia, so insurers cannot set actuarially fair premiums by detailed individual risk characteristics like unregulated classes of insurance, and this may increase the potential of adverse selection (Buchmueller, 2008). Conversely, policies designed to encourage low-risk demographic groups to purchase PHI may offset the risk of adverse selection. Given these conflicting possibilities, it is pertinent to understand whether there is evidence of adverse selection during the perinatal period as this has public policy implications and can inform risk management and pricing strategies for private health insurers. Therefore, the objectives of our study are threefold: to investigate the profiles of women by patient status (public versus private); PHI status; and transition status (for transitions between the public and private system for a subsequent pregnancy). 


\section{Methods}

\subsection{Data}

A nationally representative sample of women was randomly recruited to the Australian Longitudinal Study on Women's Health (ALSWH) through the Medicare database in 1996 and participants have since been surveyed every three to four years (Dobson et al., 2015). The Centre of Health Record and Linkage used probabilistic data linkage principles to link all available data for women residing in New South Wales (NSW) (the largest state in Australia) in the 1973-1978 cohort with the Perinatal Data Collection (PDC) for birth records and the Admitted Patients Data Collection (APDC) for hospital records. The resultant dataset used in our analysis consisted of 3,400 singleton births (from 1,875 women) over the years 2001-2012. Extensive details of this linked longitudinal data have been published elsewhere (William et al., 2018a) and detailed methods of ALSWH have also been previously published (Brown et al., 1998; Dobson et al., 2015). The PDC is a population-based surveillance system covering all births in NSW public and private hospitals, as well as homebirths, and reporting of all births in NSW to the PDC is a statutory requirement (Centre for Health Record Linkage). The APDC contains all inpatient separations (discharges, transfers and deaths) in NSW (Centre for Health Record Linkage).

A woman was defined as a "public" or "private" patient if all hospitalisations during the perinatal period were public or private respectively. Similarly, for PHI status, a woman was defined as having PHI if she had PHI for hospital treatment for all hospitalisations during the perinatal period. When examining patient and PHI status, we excluded births from women that switched between patient status during the perinatal period (a "mixed" group) and women with missing (or not stated) patient and PHI status so that each group was homogenous $(N=2,955)$. However, we included all births $(N=3,400)$ when studying the mixed group $(N=229)$. For transition status, a woman was defined as having a transition if she moved between the public and private system (or vice versa) for her second birth. We only included women for which at least the first two births were recorded in the data and excluded women with missing or not stated patient status $(N=695)$ when modelling transitions. We modelled cases of private $(N=397)$ separately to public $(N=298)$, where private and public were defined as the patient status of her second birth.

In line with previous studies, our clinical definition of "low-risk" was a non-smoking woman aged 20-34 years without pre-eclampsia or diabetes, giving birth at 37-41 weeks gestation to a foetus weighing greater than $2.5 \mathrm{~kg}$ in a cephalic presentation (Read et al., 1994; Dahlen et al., 2012). Obstetric interventions were also based on previous studies and are epidural pain relief, induction, instrumental birth (forceps or vacuum), caesarean section and episiotomy (Dahlen et al., 2012). The perinatal period was defined as the start of the antenatal period (which was defined using gestation age and date of birth of the baby) to the end of the first year following the birth of the baby and we included all hospitalisations of the women in the dataset over this period (William et al., 2018a).

\subsection{Statistical analysis}

The main purpose of the statistical analysis was to identify factors of statistical significance for public-private patients. We used the two-phase modelling methodology developed by William et al. (2018a) to account for the vast number of factors available for testing. The two phases include an exploratory phase using Classification and Regression Trees to initially select factors and a formal parametric modelling phase to assess these factors for statistical significance. In the parametric modelling phase, we used standard logistic regression using births as the unit of analysis.

Initially, over one hundred factors were available from the linked datasets to be included in the multivariate models. The factors were broadly grouped into five categories: health service use, obstetric and reproductive factors, sociodemographic factors, health behaviours and 
Table 1. Logistic regression output for single perinatal period

\begin{tabular}{|c|c|c|c|c|}
\hline Predictor & Estimate & Std. error & z-value & $p$-value \\
\hline \multicolumn{5}{|c|}{ Response $=$ patient status (private $=1$, public $=0 ; N=2,955$ ) } \\
\hline Had private health insurance & 6.58 & 0.25 & 26.17 & $<0.001$ \\
\hline Used specialist & 0.88 & 0.19 & 4.71 & $<0.001$ \\
\hline Had an intervention & 0.72 & 0.19 & 3.84 & $<0.001$ \\
\hline More access to specialists & 0.28 & 0.06 & 4.37 & $<0.001$ \\
\hline More GP use & -0.23 & 0.06 & -4.09 & $<0.001$ \\
\hline \multicolumn{5}{|c|}{ Response $=$ private health insurance status (yes $=1$, no $=0 ; N=2,955$ ) } \\
\hline Higher maternal age & 0.14 & 0.02 & 7.08 & $<0.001$ \\
\hline In low-risk category & 0.37 & 0.11 & 3.29 & 0.001 \\
\hline Had an intervention & 1.05 & 0.11 & 9.88 & $<0.001$ \\
\hline More access to specialists & 0.25 & 0.04 & -6.74 & $<0.001$ \\
\hline Partnered & 1.30 & 0.30 & 4.36 & $<0.001$ \\
\hline More education & 0.37 & 0.04 & 10.09 & $<0.001$ \\
\hline Had healthcare card & -0.65 & 0.16 & -4.12 & $<0.001$ \\
\hline More advantaged SEIFA & 0.01 & 0.00 & 7.85 & $<0.001$ \\
\hline More GP use & -0.23 & 0.03 & -6.92 & $<0.001$ \\
\hline Used specialist & 1.11 & 0.11 & 10.58 & $<0.001$ \\
\hline Higher Apgar score at 5 minutes & 0.27 & 0.06 & 4.62 & $<0.001$ \\
\hline \multicolumn{5}{|c|}{ Response $=$ mixed patient status (mixed $=1$, unmixed $=0 ; N=3,400$ ) } \\
\hline Had IVF & 1.05 & 0.28 & 3.82 & $<0.001$ \\
\hline Had private health insurance & 0.95 & 0.26 & 3.71 & $<0.001$ \\
\hline Other/not stated PHI & 3.55 & 0.27 & 13.30 & $<0.001$ \\
\hline
\end{tabular}

psychosocial and physical health factors. Factors selected for formal parametric testing following the exploratory phase are shown in the Supplementary Material and also include factors that were deemed relevant from previous studies (William et al., 2018a,b). For the transition models, we considered factors from the first and the second perinatal periods. We used a $1 \%$ significance level to ensure models were parsimonious.

\section{Results}

Frequencies of selected maternal and neonatal characteristics by patient status are shown in the Supplementary Material. The results of the logistic regressions for a single perinatal period are shown in Table 1. For the patient status model, PHI status was the major driver of whether a patient elected public or private status, even after controlling for other significant factors. We also found private patients had higher self-rated access to specialist services and were more likely to have obstetric interventions. Unsurprisingly, general practitioner use was more likely for public patients, but specialist use was more likely for private patients. Importantly, health-related factors such as physical and mental health factors, health behaviours and conditions particularly problematic for pregnancy (such as high body mass index, diabetes and hypertension) were not statistically significant. Similarly, age, parity, low-risk and sociodemographic factors were statistically insignificant; however, these factors may be captured within PHI status (and were tested therein).

Sociodemographic factors were the most dominant explanatory factors for whether a woman had PHI or not. Specifically, a woman was more likely to have PHI if she was older, partnered, 
Table 2. Logistic regression output for transition * models

\begin{tabular}{lcccc}
\hline Predictor & Estimate & Std. error & $z$-value & $p$-value \\
\hline Response $=$ transition to public patient status (transition $=1$, no transition $=0 ; N=695)$ \\
\hline Change in PHI status & 2.67 & 0.44 & 6.06 & $<0.001$ \\
\hline Response $=$ transition to private patient status & (transition $=1$, no transition $=0 ; N=695)$ \\
\hline Change in PHI status & 3.04 & 0.36 & 8.46 & $<0.001$ \\
\hline More access to specialists in first pregnancy & -0.38 & 0.11 & -3.63 & $<0.001$ \\
\hline More stress about motherhood in first pregnancy & 0.40 & 0.11 & 3.71 & $<0.001$ \\
\hline Hypertension in second pregnancy & 1.65 & 0.49 & 3.35 & $<0.001$ \\
\hline
\end{tabular}

* "Transition" identifies whether the patient status of the second birth was different to the patient status of the first birth.

more educated, with higher socioeconomic status and without a healthcare card (that is, a concession card for welfare recipients that entitles them to subsidised healthcare costs). Moreover, health-related factors outlined in the previous model were not statistically significant in this model too. Importantly, women who had PHI were more likely to be in the low-risk category and have interventions. We removed the intervention factor to test whether health-related factors became significant (as they may be highly correlated with interventions); however, they were still statistically insignificant. Those who had PHI were also more likely to have higher self-rated access to medical specialists, more likely to use specialists and less likely to use general practitioners. Finally, higher Apgar scores (at 5 minutes) were more likely for births from women with PHI and this result persisted when modelled on low-risk cases only. In summary, the results validated that there was no statistical evidence of adverse selection of PHI and that sociodemographic factors were the overriding influences for purchasing PHI in Australia.

Next we considered transitions between systems across single and multiple perinatal periods. For mixed patient status within a perinatal period, the only factor of significance besides PHI, was in-vitro fertilisation (IVF), which indicated these patients preferred (or needed) access to both systems during their perinatal period. This is possible within the Australian system because patients are able to elect to use both systems, but these results show that it is uncommon, with only $13 \%$ of patients transitioning within a perinatal period and likely for a highly specialist service.

Table 2 shows the results of the logistic regressions for transitions across two perinatal periods. Overall, we observed $10 \%$ of women transitioned between systems for their second pregnancy $8 \%$ of public patients transferred to the private system for their second baby and the equivalent figure for the public system was $2 \%$. The transition models also showed that a change in PHI status between births was the most significant predictor of whether there was a transition in patient status. This was unsurprising given earlier results showing the key driver of patient status was PHI. For the private model, a woman was also more likely to transition if she had hypertension in her second pregnancy and suggested this transition occurred due to a new health condition (as hypertension in the previous pregnancy was not significant). This factor relates to general hypertension (as opposed to pregnancy-related hypertension) so the condition could have developed in-between pregnancies. A woman was also more likely to transition to the private system if she had less self-rated access to medical specialists and greater stress about motherhood in her first perinatal period. This may indicate that women who purchased PHI in-between pregnancies did so for greater choice in healthcare providers in their next pregnancy or because of a stressful first motherhood experience. We removed the "change in PHI status" factor to test whether health-related factors became significant; however, they were still statistically insignificant (with the exception of hypertension in the second pregnancy for the private model). This is likely due to sociodemographic (rather than health-related) factors driving this change in status. 


\section{Discussion}

Our study overwhelmingly showed that the key predictor of private patient status was having PHI across both one and two perinatal periods. The decision to purchase PHI was also largely driven by sociodemographic factors, with women who were older, partnered, more educated and with higher socioeconomic status more likely to have PHI. Increased access to specialists and usage of private services were also identified as significant factors for the private setting. Furthermore, there was limited evidence to suggest that women in the private system had more complex health conditions. In contrast, women with PHI were more likely to be lower-risk and have babies with higher Apgar scores (at 5 minutes) (a measure of neonatal health) but also more likely to have an obstetric intervention.

The models for women who transitioned between systems also identified PHI status (rather than health factors) as the key driver for decision-making. For transitions within a perinatal period, IVF was also identified as significant, suggesting that women who receive IVF are using both public and private services during the perinatal period. For transitions over two perinatal periods, we found that $10 \%$ of women transitioned from one system to the other for their second perinatal period, and this was largely driven by a change in PHI status when it occurred. Hypertension was the only health condition identified as statistically significant and this was for women transitioning from public to private. This finding suggested that a new health condition may have prompted women to change systems, but as this applied to a small proportion of women it did not impact significantly on the overall drivers of patient and PHI status. The private transition model also showed that access to medical specialists and stress about motherhood were also significant factors for transitioning.

The overwhelming significance of sociodemographic factors in the private setting showed that financial penalties placed on higher income earners in Australia to purchase PHI likely outweighed the possibility of adverse selection of insurance due to community rating as health-related factors were not statistically significant. These socioeconomic disparities by public-private system for women during childbearing years are consistent with general studies that investigated income disparities by system (Hindle \& McAuley, 2004; Segal, 2004; Walker et al., 2007; Palangkaraya et al., 2009). While studies on public-private maternal health disparities generally acknowledge socioeconomic disparities (Dahlen et al., 2014; Adams et al., 2018), they do not directly address the potential for adverse selection during childbearing years largely due to the more detailed health data that is required to inform this.

The risk profiles of privately insured individuals during childbearing years warrant further consideration. We find women with PHI were lower risk, and this may be partly explained by some high-risk women being transferred to specialist units in public hospitals due to the expertise and services available in these units. Therefore, women who were at higher risk and aware of this prior to pregnancy may be less likely to purchase PHI for maternal healthcare in the first place. Moreover, our results suggest that policies introduced to encourage lower risk demographic groups into the private system have had an influence on the risk profiles observed. The few studies that consider adverse selection under current PHI regulations also find that they have been effective in improving general risk profiles of policyholders, especially through the introduction of the LHC (Buchmueller, 2008). Our study showed that these findings persist in the perinatal period, with the exception of a small proportion of women who may transition into the private system from the public system after developing hypertension. This is important from an insurer's risk management perspective, as the perinatal period is a time period during which PHI claims increase considerably (Australian Prudential Regulatory Authority, 2018) and it provides insight into appropriate risk management strategies to counteract adverse selection. Specifically, pre-existing conditions such as hypertension should be disclosed by individuals for insurance purposes and it is prudent to exclude claims on such conditions for some period of time for new policies (that is, impose a waiting period on pre-existing conditions such as hypertension). Notwithstanding these considerations, given our findings on the lower-risk profile of privately insured individuals 
overall, we determine that government policies have been effective in addressing adverse selection in $\mathrm{PHI}$ in the perinatal period.

Our results also highlighted that increased access to maternal health services and "peace of mind" may be the reasons for individuals to purchase PHI. In particular, we found the evidence that women may switch to the private system to obtain greater access to specialist services and peace of mind for a second pregnancy. However, our findings also showed that these options were largely only available to women of better socioeconomic background who were more able to pay and therefore use private services through the purchase of PHI. These findings agree with other studies that favourably value choice and control over healthcare (Natalier \& Willis, 2008; Harley et al., 2011; Willis \& Lewis, 2016).

Our findings agree with numerous previous studies that found the rates of interventions were higher in the private setting (Fisher et al., 1995; Shorten \& Shorten, 2000; Robson et al., 2009; Dahlen et al., 2012, 2014; Einarsdóttir et al., 2012, 2013b). Dahlen et al. (2014) argued that it was clinicians' behavioural differences that increased private intervention rates, even among lowrisk women, which resulted in other adverse maternal and neonatal outcomes, and ultimately increased costs incurred. Conversely, other studies have found that neonatal outcomes were better in the private system (Robson et al., 2009; Adams et al., 2017, 2018; Jang et al., 2017). In particular, Adams et al. (2018) control for a vast number of risk factors and conclude that clinical practices may be partly responsible for lower neonatal mortality rates in the private system. Given our findings show limited evidence of adverse selection in the private system but higher rates of interventions, our findings agree with conclusions that there are disparities in clinical practices by system. We recommend further research in this area to understand these interactions better.

The strengths of this study (in contrast to previous studies) are the extensive number of sociodemographic and health-related factors that we were able to test within a multivariate model framework because of the comprehensive linked cohort data used to determine the extent of adverse selection in maternal healthcare. The longitudinal data also allowed us to analyse women across two perinatal periods to study reasons for transitioning between systems for the first time. On the other hand, the main limitations of the study were the smaller-sized dataset for women in one state only (NSW) and a predominantly low-risk cohort of women. Also, although ALSWH recruited a nationally representative sample, there was an over-representation of women with tertiary education and an under-representation of women from non-English-speaking backgrounds (Dobson et al., 2015). There was also the possibility of attrition bias; however, previous analyses have found that this bias was unlikely to be significant (Powers \& Loxton, 2010).

Finally, we note that recent trends show a slight reduction in the proportion of the population with PHI for hospital treatment and a corresponding reduction in the use of private maternal healthcare services in Australia (Australian Institute of Health and Welfare, 2017a, 2017b, 2018). Although it is too early to determine whether this trend will continue or have a significant impact on the risk profile of patients, the results of our study overwhelmingly show limited evidence for adverse selection and it is unlikely that the small movements observed to date will significantly impact on this finding. Furthermore, there are new reforms to the PHI industry (The Department of Health, 2018b) that aim to make it simpler and more affordable. It will take a number of years to implement these new reforms but recommended initiatives should continue to counteract the effect of adverse selection. These reforms will apply in addition to the regulations that are currently in place. We recommend that these issues are revisited once the new policies are in place.

\section{Conclusion}

When a mixed public-private maternal health system is within a regulatory structure that incentivises specific groups to purchase PHI, sociodemographic factors overwhelmingly influenced decision-making into each system. While it was uncommon to switch between systems, the main factor in transitioning to the private sector for a subsequent pregnancy was change in PHI status. Previous stress about motherhood, previous self-rated access to specialist services, and 
hypertension were also found to be statistically significant factors for transitioning systems. As the statistical significance of hypertension related only to the small number of cases that transitioned to the private system and health-related conditions were not statistically significant predictors of overall patient or PHI status, we determine that there was limited evidence of adverse selection. We conclude that targeted government regulations in PHI have effectively reduced adverse selection even during the perinatal period of policyholders.

\section{Ethics}

The ALSWH has been granted ethics clearance by the Universities of Newcastle and the University of Queensland (Ethics approvals H0760795 and 2004000224). Ethics for the longitudinal study was approved on 26 July 1995. This research also received ethical approval from the Australian National University (Protocol: 2017/106).

Author ORCID. (D) Jananie William, https://orcid.org/0000-0003-1986-985X

Supplementary Material. To view supplementary material for this article, please visit https://doi.org/10.1017/ S1748499519000083

Acknowledgements. The research on which this study is based was conducted as part of the ALSWH by the University of Queensland and the University of Newcastle. We are grateful to the Australian Government Department of Health for funding and to the women who provided the survey data. We acknowledge the Australian Institute of Health and Welfare as the integrating authority for these data. We thank the NSW Ministry of Health, the NSW Central Cancer Registry and the Centre for Health Record Linkage.

\section{References}

Adams, N., Gibbons, K. S. \& Tudehope, D. (2017). Public-private differences in short-term neonatal outcomes following birth by prelabour caesarean section at early and full term. Australia \& New Zealand Journal of Obstetrics and Gynaecology, 57(2), 176-185.

Adams, N., Tudehope, D., Gibbons, K. \& Flenady, V. (2018). Perinatal mortality disparities between public care and private obstetrician-led care: a propensity score analysis. BJOG: An International Journal of Obstetrics \& Gynaecology, 125(2), 149-158.

Australian Institute of Health and Welfare. (2017a). Australia's mothers and babies 2015 - in brief. Perinatal Statistics Series no. 33. Cat no. PER 91. Canberra: AIHW.

Australian Institute of Health and Welfare. (2017b). Private health insurance use in Australian hospitals, 2006-07 to 2015-16: Australian hospital statistics. Health Services Series no. 81. Cat. no. HSE 196. Canberra: AIHW.

Australian Institute of Health and Welfare. (2018). Australia's mothers and babies 2016 - in brief. Perinatal Statistics Series no. 34. Cat. no. PER 97. Canberra: AIHW.

Australian Prudential Regulatory Authority. (2018). Private Health Insurance Quarterly Statistics. Australian Prudential Regulatory Authority, Sydney.

Australian Taxation Office. (2018a). Medicare levy surcharge. Available online at the address https://www.ato.gov.au/ Individuals/Medicare-levy/Medicare-levy-surcharge/ [09-Feb-2018].

Australian Taxation Office. (2018b). Private health insurance rebate. Available online at the address https://www.ato.gov.au/ Individuals/Medicare-levy/Private-health-insurance-rebate/ [accessed 13-Feb-2018].

Brown, W., Bryson, L., Byles, J., Dobson, A., Lee, C., Mishra, G. \& Schofield, M. (1998). Women's Health Australia: recruitment for a national longitudinal cohort study. Women Health, 28, 23-40.

Buchmueller, T. (2008). Community rating, entry-age rating and adverse selection in private health insurance in Australia. The Geneva Papers on Risk and Insurance - Issues and Practice, 33(4), 588-609.

Centre for Health Record Linkage. Data dictionaries for Centre for Health Record Linkage. Available online at the address http://www.cherel.org.au/data-dictionaries [accessed Nov 2016].

Dahlen, H., Tracy, S., Tracy, M., Bisits, A., Brown, C. \& Thornton, C. (2012). Rates of obstetric intervention among lowrisk women giving birth in private and public hospitals in NSW: a population-based descriptive study. BMJ Open, 2. doi: e001723

Dahlen, H., Tracy, S., Tracy, M., Bisits, A., Brown, C. \& Thornton, C. (2014). Rates of obstetric intervention and associated perinatal mortality and morbidity among low-risk women giving birth in private and public hospitals in NSW (20002008): a linked data population-based cohort study. BMJ Open, 4(e004551). doi: e001723

Dobson, A. J., Hockey, R., Brown, W. J., Byles, J. E., Loxton, D. J., McLaughlin, D. \& Mishra, G. D. (2015). Cohort profile update: Australian Longitudinal Study on Women's Health. International Journal of Epidemiology, 44(5), 1547a-1547f. 
Einarsdóttir, K., Haggar, F., Langridge, A. T., Gunnell, A. S., Leonard, H. \& Stanley, F. J. (2013a). Neonatal outcomes after preterm birth by mothers' health insurance status at birth: a retrospective cohort study. BMC Health Services Research, 13,40 .

Einarsdóttir, K., Haggar, F., Pereira, G., Leonard, H., de Klerk, N., Stanley, F. J. \& Stock, S. (2013b). Role of public and private funding in the rising caesarean section rate: a cohort study. BMJ Open, 3(5), e002789.

Einarsdóttir, K., Kemp, A., Haggar, F. A., Moorin, E. M., Gunnell, A. S., Preen, D. B., Holman, C. D. (2012). Increase in caesarean deliveries after the Australian Private Health Insurance Incentive Policy Reforms. PLoS One, 7(7).

Einarsdóttir, K., Stock, S., Haggar, F., Hammond, G., Langridge, A. T., Preen, D. B., Stanley, F. J. (2013c). Neonatal complications in public and private patients: a retrospective cohort study. BMJ Open, 3(5), e002786.

Fisher, J., Smith, A. \& Astbury, J. (1995). Private health insurance and a healthy personality: new risk factors for obstetric intervention? Psychosomatic Obstetrics \& Gynecology, 16(1), 1-9.

Harley, K., Willis, K., Gabe, J., Short, S. D., Collyer, F., Natalier, K. \& Calnan, M. (2011). Constructing health consumers: private health insurance discourses in Australia and the United Kingdom. Health Sociology Review, 20(3), 306-320.

Hindle, D. \& McAuley, I. (2004). The effects of increased private health insurance: a review of the evidence. Australian Health Review, 28(1), 119-138.

Hoy, M. \& Lun, E. (2017). Adverse selection and insurance. In eLS, John Wiley \& Sons. doi: 10.1002/9780470015902.a0026687

Jang, W., Flatley, C., Greer, R. M. \& Kumar, S. (2017). Comparison between public and private sectors of care and disparities in adverse neonatal outcomes following emergency intrapartum cesarean at term - a retrospective cohort study. PLoS One, 12(11), e0187040.

Natalier, K. \& Willis, K. (2008). Taking responsibility or averting risk? A socio-cultural approach to risk and trust in private health insurance decisions. Health, Risk \& Society, 10(4), 399-411. doi: 10.1080/13698570802167413

Nippita, T., Lee, Y., Patterson, J., Ford, J., Morris, J., Nicholl, M. \& Roberts, C. (2015). Variation in hospital caesarean section rates and obstetric outcomes among nulliparae at term: a population-based cohort study. BJOG: An International Journal of Obstetrics \& Gynaecology, 122(5), 702-711.

Palangkaraya, A., Yong, J., Webster, E. \& Dawkins, P. (2009). The income distributive implications of recent private health insurance policy reforms in Australia. European Journal of Health Economics, 10(2), 135-148.

Powers, J. \& Loxton, D. (2010). The impact of attrition in an 11-year prospective longitudinal study of younger women. Annals of Epidemiology, 20, 318-321.

Read, A., Prendiville, W., Dawes, V. \& Stanley, F. (1994). Cesarean section and operative vaginal delivery in low-risk primiparous women, Western Australia. American Journal of Public Health, 84(1), 37-42.

Roberts, C. L., Tracy, S. \& Peat, B. (2000). Rates for obstetric intervention among private and public patients in Australia: population based descriptive study. British Medical Journal, 321(7254), 137-141.

Robson, S., Laws, P. \& Sullivan, E. (2009). Adverse outcomes of labour in public and private hospitals in Australia: a population-based descriptive study. The Medical Journal of Australia, 190(9), 474-477.

Segal, L. (2004). Why it is time to review the role of private health insurance in Australia. Australian Health Review, 27(1), 3-15.

Shorten, A. \& Shorten, B. (2000). Women's choice? The impact of private health insurance on episiotomy rates in Australian hospitals. Midwifery, 16(3), 202-212.

Shorten, A. \& Shorten, B. (2002). Perineal outcomes in NSW public and private hospitals: analysing recent trends. Australian Journal of Midwifery, 15(2), 5-10.

The Department of Health. (2018a). Lifetime Health Cover. Available online at the address http://www.health.gov.au/ internet/main/publishing.nsf/content/health-privatehealth-lhc-providers-general.htm [accessed 9-Feb-2018].

The Department of Health. (2018b). Private Health Insurance Reform Rules 2018. Available online at the address https:// www.health.gov.au/internet/main/publishing.nsf/Content/private-health-insurance-reform-rules-2018 [accessed 10-Jan2018].

Thomas, G. (2017). Loss Coverage: Why Insurance Works Better with Some Adverse Selection. Cambridge University Press, Cambridge.

Walker, A. E., Percival, R., Thurecht, L. \& Pearse, J. (2007). Public policy and private health insurance: distributional impact on public and private hospital usage. Australian Health Review, 31(2), 305-314.

William, J., Chojenta, C., Martin, M. \& Loxton, D. (2018a). An actuarial investigation into maternal hospital cost risk factors for public patients. Annals of Actuarial Science, 12(1), 106-129.

William, J., Chojenta, C., Martin, M. \& Loxton, D. (2018b). An actuarial investigation into maternal out-of-hospital cost risk factors. Annals of Actuarial Science, 1-35.

Willis, K. \& Lewis, S. (Producer). (2016). Which are better, public or private hospitals? The Conversation. Available online at the address https://theconversation.com/which-are-better-public-or-private-hospitals-54338 [accessed 9-Feb-2018].

Cite this article: William J, Loong B, Chojenta C and Loxton D (2019). The profiles of public and private patients in maternal healthcare: a longitudinal study to examine adverse selection. Annals of Actuarial Science, 1-9. https://doi.org/10.1017/ S1748499519000083 